\title{
Screening for Duchenne muscular dystrophy
}

\author{
J Fenton-May, D M Bradley, J R Sibert, R Smith, E P Parsons, P S Harper, A Clarke
}

Diagnostic delay has long been recognised as a major problem for families with boys affected by Duchenne muscular dystrophy, both because the families may have further affected boys in ignorance of the elder child's disease ${ }^{1}$ and because the protracted diagnostic delay is itself distressing. ${ }^{2}{ }^{3}$ Although screening of newborn infants has been technically possible since $1975,{ }^{4}$ fears were expressed that it might generate greater distress than is experienced at a later diagnosis. It was therefore suggested that screening of boys not walking independently by 18 months of age could help to reduce the unacceptably long diagnostic delays and would be more acceptable to families. ${ }^{56}$

This type of screening programme, however, would not identify two groups of affected boys: (a) those who are walking at 18 months (Gardner-Medwin et al report that $50 \%$ of boys with Duchenne muscular dystrophy have begun to walk independently by the age of 18 months ${ }^{5}$ ); and (b) those non-walking boys from whom a blood spot for creatine kinase analysis is not obtained, either as a logistic failure or because the parents decline the offer of the test.

\section{Screening of non-walking boys at 18 months of age}

For 18 months from January 1986 we implemented and evaluated a programme of blood spot creatine kinase screening for boys in Wales who were not able to take four steps independently at 18 months of age; at the time of this study, health districts in Wales had a programme of screening development in all children at 18 months using the Denver test. There were 25229 boys eligible for Denver assessment during this period, of whom $79 \%$ were assessed. Of those assessed, $1.7 \%$ were not walking independently, and of the nonwalking boys $61 \%$ had a blood spot taken for creatine kinase analysis. ${ }^{7}$

\section{Follow up to 18 month screening}

Duchenne muscular dystrophy was diagnosed in two boys eligible for Denver assessment during the 18 month period of screening who were walking at that time. One boy walked from 15 to 16 months, but was referred for orthopaedic assessment at 2 years because of family concern about his flat feet and knock knees. He was subsequently noticed to be clumsy and to have difficulty in climbing stairs and was referred for paediatric assessment at 4 years. Investigations showed a grossly increased serum creatine kinase concentration and muscle pathology (including dystrophin immunocytochemistry) that was diagnostic of Duchenne muscular dystrophy.

The second boy was late sitting, but walked at 12 months. He was considered to be clumsy and to have speech delay, and concern was voiced by his nursery school; this led to paediatric referral at $4 \cdot 1$ years. Investigation showed a grossly increased serum creatine kinase concentration, a molecular deletion within the Duchenne muscular dystrophy gene, and muscle pathology diagnostic of Duchenne muscular dystrophy.

These two boys bring to seven the total of affected boys eligible for screening during the study period. Two were identified by the screening programme. Three were identified before 18 months of age, one presenting at 10 months with failure to thrive, and one at 17 months with 'late walking'; the third was diagnosed early because of a family history. The final two boys are reported here. This brings the birth incidence of Duchenne muscular dystrophy in this cohort to 1 in 3600; the 18 month screening programme identified only two of the seven affected boys.

\section{Screening of newborn infants}

During the screening programme of 18 month old boys it became clear that less than $50 \%$ of non-walking boys were being screened $(61 \%$ of the non-walkers from $79 \%$ of the eligible cohort is $48 \%$ ), despite considerable effort by the project team. Previous research ${ }^{5}$ reporting that $50 \%$ of affected boys would be walking at this stage only compounded the problem because it meant that we could expect to detect only $25 \%$ of boys with Duchenne muscular dystrophy. This led us to reconsider the case for screening of newborn infants. There was no empirical evidence to suggest that screening of newborn infants would cause any greater distress than a later traditional diagnosis, but clearly any such programme would have to operate on the basis of informed parental consent, and would have to incorporate a full psychosocial evaluation of the impact of such early diagnosis on the families.

It was already clear that many of the parents of affected boys favoured a screening programme within the first three months of life ( $81 \%$ of parents in the study of Firth et al
Department of Health, York District R Smith

Correspondence to: Dr Clarke. 
would have preferred screening at that stage ${ }^{3}$ and an attitude survey conducted among mothers of newborn infants in Cardiff suggested that most parents $(94 \%)$ would accept newborn screening for Duchenne muscular dystrophy, ${ }^{8}$ a figure confirmed in practice by the $95 \%$ uptake rate. ${ }^{9}$

Our preliminary experience with screening of newborn infants for Duchenne muscular dystrophy in Wales has been described, ${ }^{9}$ and shows that such a programme is technically feasible, can operate as an additional test on the basis of informed parental consent, and is acceptable to most families. It is important, however, to appreciate that the relative success of the programme to date has depended on four factors. Firstly, the research team has carefully coordinated the initial approach to each identified family with the primary health care team and the local paediatrician. Secondly, the team has ensured that each identified family has received continuing support from the appropriate health professionals. Thirdly, the programme has been designed to ensure that parental choice is maximised at each stage in the diagnostic process. Fourthly, we have been developing an educational intervention for midwives and health visitors which is aimed at increasing their knowledge about the disease and their understanding of the programme and its ethos. We have recognised the important role these health professionals have in offering the test and facilitating genuine informed parental consent.

\section{Further evaluation}

Although the preliminary experience in Wales has been positive, it would be premature to recommend that screening of newborn infants is established as a routine service programme at this stage. It is important to continue the programme on a research basis for several reasons.

- The psychosocial evaluation needs to monitor the response of families as a cohort of boys becomes symptomatic.

- The reproductive patterning of the first cohort is still being established.

- The developmental follow up of the boys has not been completed.

- There has been no evaluation of the programme from the perspective of health economics. This would be helpful for any health authority contemplating the introduction of such a screening programme into regular practice.

- Most importantly, there is a danger that the safeguards introduced into the Welsh scheme could be lost if there was a hasty transfer from research to service or if the scheme was introduced into other regions as a laboratory service initiative without the educational, coordinating, and supportive elements that are integral and essential components of the research programme. This point has been made by Statham et al ${ }^{10}$ and by Bowman. ${ }^{11}$

- One further safeguard that we will be developing over the next three years is the introduction and assessment of refinements to the process of obtaining parental consent for entry into the programme. The success of this process will have major implications for the introduction of screening of newborn infants for Duchenne muscular dystrophy on a wider scale in Britain and elsewhere.

Further reports of the experience in Wales are in preparation. One preliminary finding that can be reported is that eight of the nine boys with Duchenne muscular dystrophy who have reached 18 months were already walking independently by that age. This emphasises the importance of the continued developmental monitoring of the patients diagnosed in early infancy, and raises the possibility that early physiotherapy may have benefits for these boys.

1 O'Brien T, Sibert JR, Harper PS. Implications of diagnostic delay in Duchenne muscular dystrophy. BMF 1983; 287 1106-7.

2 Firth M. Diagnosis of Duchenne muscular dystrophy: experiences of parents of sufferers. $B M \mathcal{F} 1983 ; 286$ 700-1.

3 Firth M, Gardner-Medwin D, Hosking G, Wilkinson E. Interviews with parents of boys suffering from Duchenne muscular dystrophy. Dev Med Child Neurol 1983; 25: 466-71.

4 Zellweger H, Antonik A. Newborn screening for Duchenne muscular dystrophy. Pediatrics 1975 ; 55: $30-4$.

5 Gardner-Medwin D, Bundey S, Green S. Early diagnosis of Duchenne muscular dystrophy. Lancet 1978; i: 1102.

6 Gardner-Medwin D. Recognising and preventing Duchenne muscular dystrophy [editorial]. BMF 1983, 287: $1083-4$.

7 Smith RA, Rogers M, Bradley DM, Sibert JR, Harper PS Screening for Duchenne muscular dystrophy. Arch Dis Child 1989; 64: 1017-21.

8 Smith RA, Williams DK, Sibert JR, Harper PS. Attitudes of mothers to neonatal screening for Duchenne muscular dystrophy. BMF 1990; 300: 1112.

9 Bradley DM, Parsons EP, Clarke A. Experience with screening newborns for Duchenne muscular dystrophy in Screening newborns for Duchenn

10 Statham H, Green J, Snowdon C. Mothers' consent to screening newborn babies for disease. BMF 1993; 306: 858-9.

11 Bowman E. Screening newborn infants for Duchenne muscular dystrophy. BMF 1993; 306: 349 . 